\title{
Creation of Highly Ordered "Nano-Mille-Feuille" Hard/Soft Nanoparticle Multilayers with Inter-Particle Cross-Linking by Diacetylene-Containing Chains
}

\author{
Takato Ohashi, ${ }^{a}$ Nanata Kikuchi, ${ }^{\mathrm{b}}$ and Atsuhiro Fujimori ${ }^{\mathrm{a}, *}$
}

${ }^{\mathrm{a}}$ Graduate School of Science and Engineering, and ${ }^{\mathrm{b}}$ Faculty of Engineering, Saitama University, 255 Shimo-okubo, Sakura-ku, Saitama 338-8570, Japan

*Corresponding Author

Tel and Fax: +81-48-858-3503

E-mail address: fujimori@apc.saitama-u.ac.jp

Keywords: Polymer Nanosphere, Organo-Modified Inorganic Nanoparticles, Alternating Multi-Particle Layers, Photo-crosslinking by Diacetylene 


\section{[Figure Caption]}

Figure S1. Schematic illustrations of research strategy in this study.

Figure S2. Schematic diagram of the comprehensive conclusions in this study. 


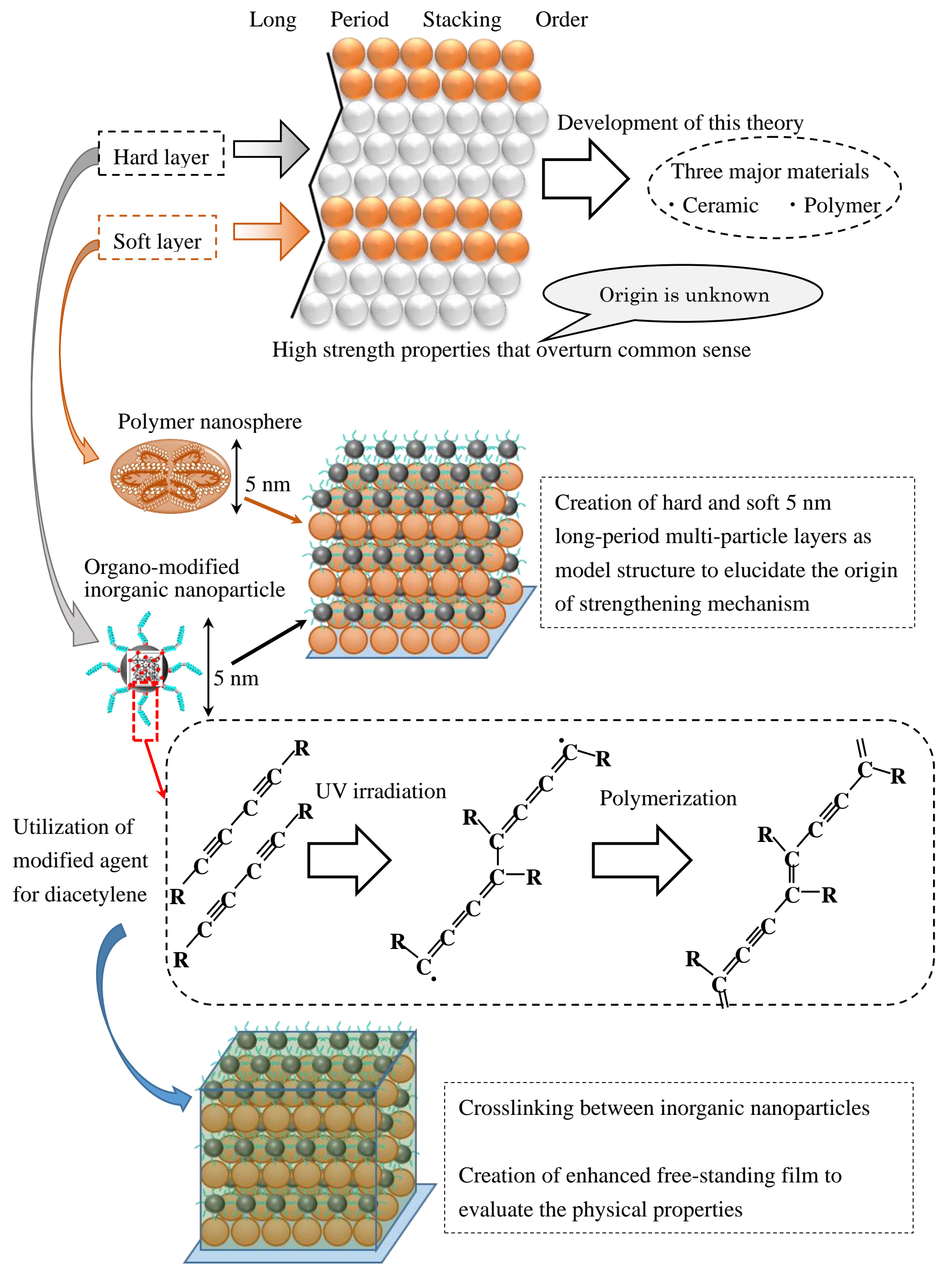

Figure S1

T. Ohashi, et al 


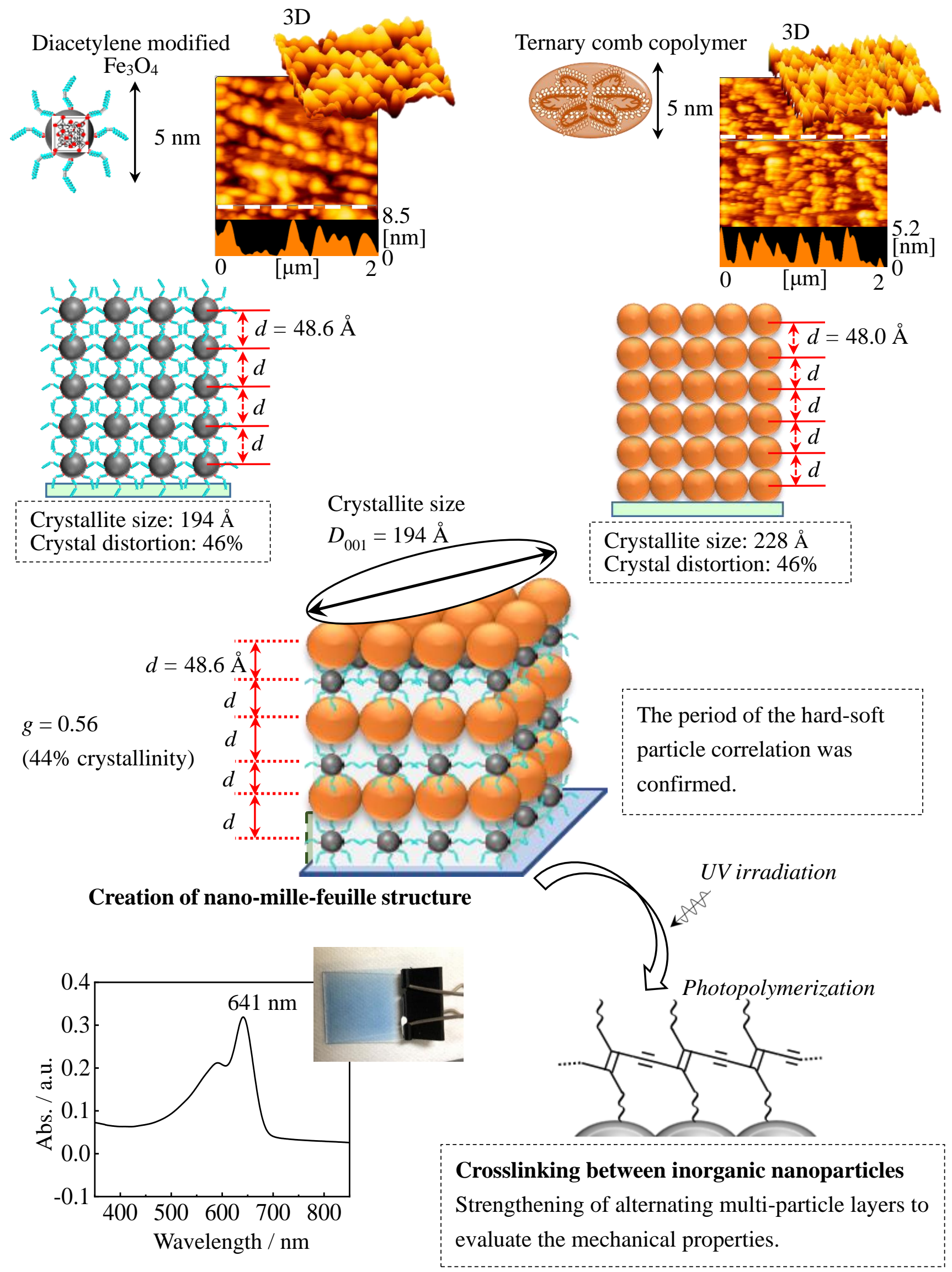

Figure S2

T. Ohashi, et al 